\title{
A Community-enabled Readiness for first 1000 Days Learning Ecosystem (CRADLE) for first-time families: study protocol of a three-arm randomised controlled trial
}

See Ling Loy ${ }^{1,2,3^{*}}$ (D), Thilagamangai ${ }^{4}$, Joyce Teo ${ }^{5}$, Sze Wern Chan ${ }^{4}$, Nurul Khairani Abdul Razak ${ }^{4}$, Oh. Moh Chay ${ }^{2,6,7,8}$ and Kee Chong $\mathrm{Ng}^{2,5}$

\begin{abstract}
Background: Enhanced parenting self-efficacy (PSE) contributes to positive parenting and future parental-child health. First-time parents, in particular, are in need of support since the pregnancy until post-delivery to strengthen their early PSE. However, there is a lack of effective and sustainable relevant programmes in the community. The Community-enabled Readiness for first 1000 Days Learning Ecosystem (CRADLE) aims to develop a self-learning eco-community throughout the pregnancy and early childhood to promote PSE among first-time parents. We apply choice architecture strategy using behavioural nudges and midwife-led continuity care during the first 1000 days, and test their effects on PSE and mother-child health for first-time families in Singapore.

Methods: This three-arm randomised controlled trial will recruit up to 750 pregnant women from the KK Women's and Children's Hospital, Singapore. Participants will be randomly assigned to receive: (1) standard routine care; (2) behavioural nudges (text messages) along with the use of a social media platform; or (3) midwife-led continuity care involving individualised teleconferencing sessions, during pregnancy and post-delivery. Using web-based questionnaires, participants will be assessed for baseline socio-demography and health status in the first visit, with follow-up assessments in the third trimester, at birth, 6-week (primary end-point), 6-, 12-, 18- and 24-month postdelivery. The primary outcome is PSE. Secondary outcomes include health and birth experience, mental wellness, feeding practice, maternal and child nutritional status. Intention-to-treat and per-protocol analyses will be performed using general linear models to test the effects of interventions across three arms. Recruitment has begun in June 2020 and is estimated to complete in September 2022.
\end{abstract}

Discussion: This study may identify a sustainable effective strategy in the community by helping first-time parents to have a positive experience during the pregnancy, childbirth and parenthood, leading to an enhanced PSE and health outcomes for both mother and child. Findings from this study will provide insight into the implementation of early parenting and mother-child care programmes.

(Continued on next page)

\footnotetext{
* Correspondence: loy.see.ling@kkh.com.sg

'Department of Reproductive Medicine, KK Women's and Children's Hospital,

100 Bukit Timah Road, Singapore 229899, Singapore

${ }^{2}$ Duke-NUS Medical School, 8 College Road, Singapore 169857, Singapore

Full list of author information is available at the end of the article
}

C C The Author(s). 2021 Open Access This article is licensed under a Creative Commons Attribution 4.0 International License, which permits use, sharing, adaptation, distribution and reproduction in any medium or format, as long as you give appropriate credit to the original author(s) and the source, provide a link to the Creative Commons licence, and indicate if changes were made. The images or other third party material in this article are included in the article's Creative Commons licence, unless indicated otherwise in a credit line to the material. If material is not included in the article's Creative Commons licence and your intended use is not permitted by statutory regulation or exceeds the permitted use, you will need to obtain permission directly from the copyright holder. To view a copy of this licence, visit http://creativecommons.org/licenses/by/4.0/ The Creative Commons Public Domain Dedication waiver (http://creativecommons.org/publicdomain/zero/1.0/) applies to the data made available in this article, unless otherwise stated in a credit line to the data. 
(Continued from previous page)

Trial registration: ClinicalTrials.gov NCT04275765. Registered on 19 February 2020.

Keywords: Choice architecture, First-time parent, Midwife, Nudge, Randomised controlled trial, Self-efficacy

\section{Background}

The "Developmental Origins of Health and Disease $(\mathrm{DOHaD})$ " paradigm underlies the influences of earlylife environment on health and disease development over the lifespan $[1,2]$. Strong evidence in humans have shown optimal nurturing during pregnancy and after delivery for both mother and child, which are critically important to build an optimal foundation for subsequent long-term maternal-child health [3, 4]. These involve ensuring an optimal psychological and physical health across the pregnancy, lactation and early childhood, as covered by the window of the first 1000 days of life, i.e. 280 days of gestation plus 730 days of the first two postnatal years [5]. The $\mathrm{DOHaD}$ or the first 1000 days concept is not just about academic science, but also about education, policy making and everyday practice for individuals [3]. However, to date, most recommendations for pregnant women and young children have yet to take long-term health consequences of early life condition into account [4].

Mental wellness and nutrition, serve as the key components in the psychological and physical health. With the onset of pregnancy, there is a great need to ensure that expectant parents are mentally and physically well prepared to cope with the developing pregnancy, the childbirth and the new life with their babies [6]. On top of building a nurturing environment, this paves the way to promote and enhance early parenting self-efficacy (PSE), as evidenced by the positive link between prenatal mental wellbeing and PSE [7]. PSE, defined as parents' belief in their ability to perform the parenting role successfully [8], has emerged as an important clinical target for intervention due to its impact on parent and child wellbeing [9]. High PSE is related to effective parenting [10] and great parental competency [11]; low PSE appears to be a salient risk factor of parental depression $[12,13]$ and is closely linked to long-term harm in child physical and mental development [14-16]. Improving early PSE is thus important towards building a healthy environment for children and for parents, promoting long-term positive health outcomes.

Bandura summarised several factors that could impinge on PSE, including the lack of social support, parental depression, and temperamentally difficult infant and child health problems [17]. This is particularly relevant to first-time families where low PSE is often seen among first-time parents [18]. Specifically, first-time mothers tend to experience difficulties and are vulnerable to emotional distress due to the uncertainties, maladjustments and new challenges faced, including antenatal requirements/demands, intense lifestyle changes, adaptation as a new parent, physical and financial burden, during both pregnancy and postpartum periods [19-21]. The lack of experience, knowledge and skills in antenatal care, childbirth, breastfeeding, infant care and coping strategies have placed them in great needs to acquire social and professional supports $[6,22$, 23]. This highlights the importance of having an engaged effective support for these first-time mothers since the onset of pregnancy to promote their self-efficacy in parenting.

In this digital era, though first-time expectant parents could receive much information via the internet, parental education offered by health care professionals remains relevant due to the need of a trustworthy source of information that can be relied on [24]. Delivery of education through midwives during the antenatal and postpartum periods has shown to be useful, making those parents felt supported and prepared for parenting [24-26], with an increased PSE reported [27]. As reviewed, midwife-led continuity care starting from pregnancy until early parenting period has been associated with positive maternal outcomes and parents' satisfaction [28]. Importantly, the resulted positive maternal outcomes had contributed to reduced health care cost [29]. Unfortunately, in Singapore, there is no midwifeled continuity care service to help first-time parents in gaining positive parenting experience and building PSE. To scale up the service to make it accessible to people, community-based midwifery care is required.

A simple, cost-effective approach which has shown positive effect on parenting is the provision of parental education by health care professionals through digital platforms. A recent web-based psychoeducational intervention over 6 months post-delivery has shown to improve PSE, reduce postnatal depression and enhance social support among first-time mothers in Singapore [30]. A text messaging programme over 1 year for parents of pre-schoolers in the United States was found to be effective in supporting positive parenting practices [31]. The text messages serve as a powerful nudging tool by targeting behavioural barriers to good parenting [32]. Through messages, the complexity of parenting was broken down into small steps that are easy-to-achieve, along with continuous reinforcement and encouragement to help parents change their parenting behaviours 
and practices [33]. The use of nudges represents a choice architecture strategy which is widely used in public policy making, to alter people's behaviour and influence decision making [33-35].

\section{Aims}

The main goal of the Community-enabled Readiness for first 1000 Days Learning Ecosystem (CRADLE) study is to promote and sustain healthy behaviour both for the mother and offspring in the first 1000 days and beyond, through a supportive community-led sharing environment for first-time families. To achieve the goal, we developed a virtual interactive and sharing platform, integrated with choice architecture using calibrated nudges, along with midwife-led care service. Our target is to create a self-learning ecosystem by providing knowledge support and guidance, via interactions with health care professionals and peer learning through e-platform. This will in turn create opportunities for the participants to become empowered and eventually develop as community volunteers for maternity care.

Specifically, we aim to examine the effects on PSE and health outcomes in mother and child, with the use of behavioural nudges in the form of text messages (a choice architecture technique) and midwife-led care service, from pregnancy until the first 2 years post-delivery. We hypothesise that during the first 1000 days, the use of virtual nudging tool in the form of text messages supplemented with social media, or physical care from midwives supplemented with individualised teleconferencing sessions will lead to a better maternal PSE, health and birth experience, mental wellness, and nutritional outcomes in both mother-child, compared with those who are receiving standard routine care. The framework of the study is shown in Fig. 1.

\section{Methods/design}

\section{Trial design}

CRADLE is conceptualised as a three-arm, parallelgroup, non-blinded randomised control trial (RCT). The allocation ratio of the control group to two intervention groups is 1:1:1. The three arms comprise: (1) standard routine care; (2) behavioural nudges (text messages) along with the use of a social media platform; (3) midwife-led continuity care involving individualised teleconferencing sessions. The protocol has followed the SPIRIT guidelines (see Additional file 1). The trial registration number of the study is ClinicalTrials.gov NCT04275765.

The flow of the trial is shown in Fig. 2. Following informed consent at the recruitment visit, a baseline assessment on maternal sociodemographic characteristics and health status will be conducted, followed by randomisation to the control or intervention group (Arm 2

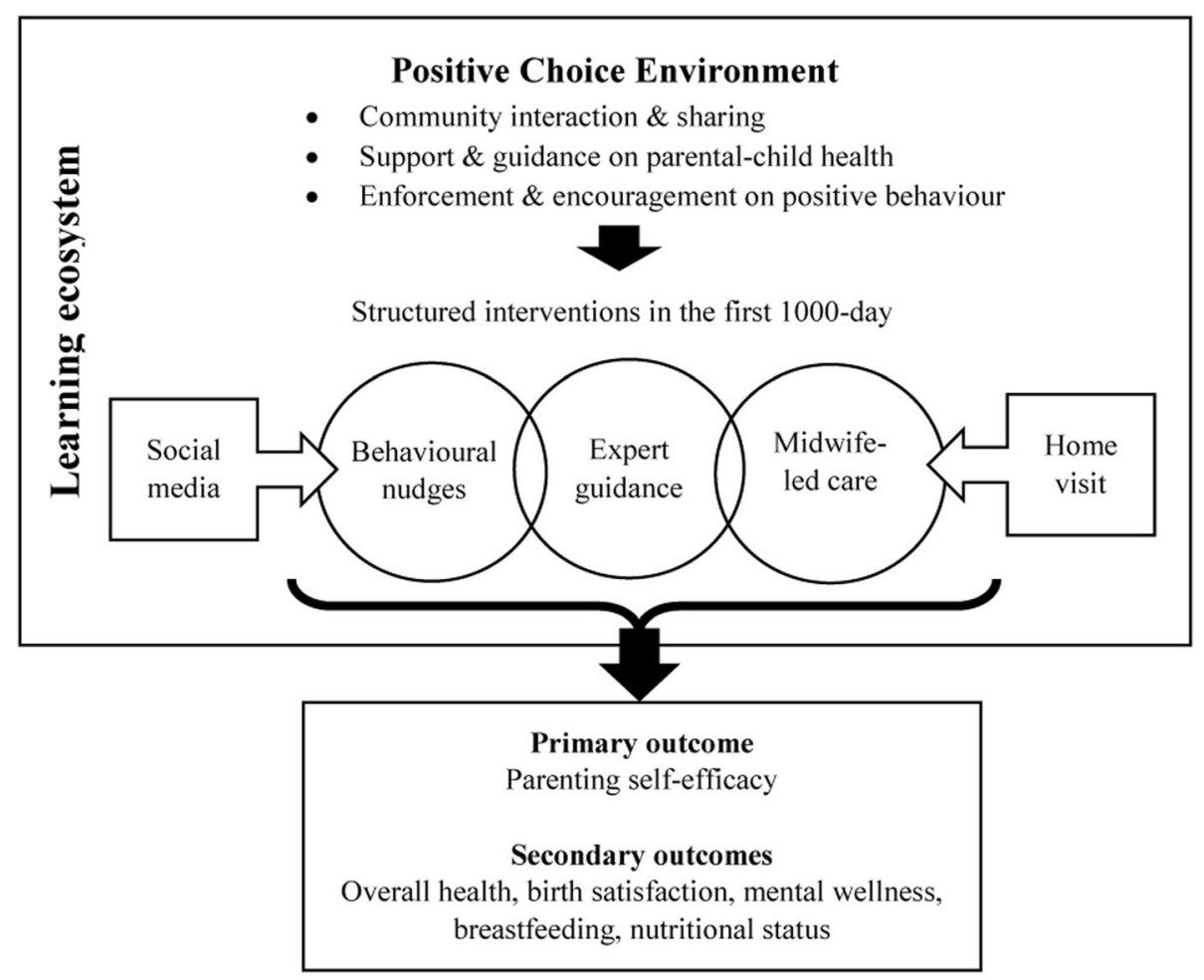

Fig. 1 Study framework 


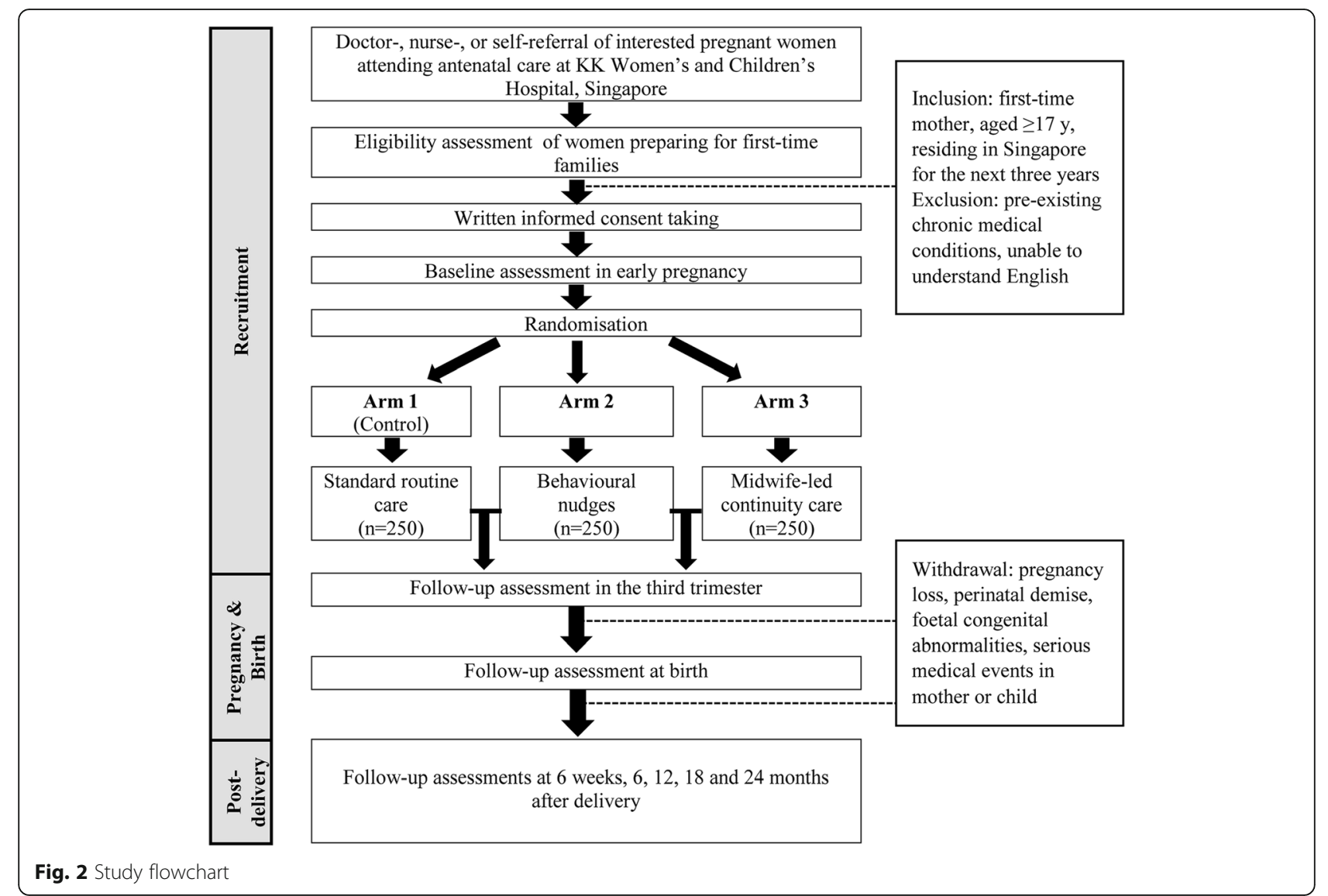

or 3) using the electronic randomisation list. Depending on the follow-up time-point (i.e. 30-week, at birth, 6week, 6-, 12-, 18-, and 24-month post-delivery), every participant is required to complete few sets of online questionnaires for assessments on PSE, health and birth experience, mental wellness and feeding practice. A 2week timeframe is given to the participants to complete the questionnaires, depending on their preferable schedule and availability within the period. Maternal and child anthropometric measures including weight and height are also assessed. Details of study measures and questionnaires used according to the visit time points are presented in Table 1.

\section{Recruitment}

Recruitment is conducted at the KK Women's and Children's Hospital (KKH), Singapore. In this study, we target to recruit up to 750 pregnant women visiting antenatal clinics of $\mathrm{KKH}$ during their early pregnancy. Recruitment brochures, posters and banners that contain general information and selection criteria of the study are placed at different locations, such as hospital lifts and clinics. Interested women self-contact the study team by email or phone, or to be referred by doctors or nurses.
Inclusion criteria are women who meet the following: first-time pregnant mother, aged 17 years and above, going to reside in Singapore for the next 3 years, able to understand English (or with a family member who is able to assist), and to provide written, informed consent. Exclusion criteria are women with medical conditions such as serious chronic diseases and high risk complicated pregnancies. Throughout the study period, participants who experience loss of pregnancy, perinatal demise, congenital abnormalities in offspring, serious medical events in mother/child, unable to comply with study protocol or wish to discontinue their participation will be withdrawn from the study. For participants who withdraw from the study, consent will be sought to retain and analyse their data until the withdrawal timepoint.

\section{The intervention}

Arm 2 and Arm 3 interventions are designed based on the framework that can eventually lead to a self-learning ecosystem in the community to improve early PSE and health outcomes for first-time families.

In Arm 2, we interact with the participants virtually. The participants in this intervention arm will receive weekly mobile messages on important information 
Table 1 Data collection and study instruments

\begin{tabular}{|c|c|c|c|c|c|c|c|c|}
\hline & Baseline visit & $\begin{array}{l}\text { Third } \\
\text { trimester }\end{array}$ & Birth & 6 weeks & 6 months & 12 months & 18 months & 24 months \\
\hline \multicolumn{9}{|l|}{ Socio-demography } \\
\hline Age & $\sqrt{ }$ & & & & & & & \\
\hline Ethnicity & $\sqrt{ }$ & & & & & & & \\
\hline Family structure & $\sqrt{ }$ & & & & & & & \\
\hline Education & $\sqrt{ }$ & & & & & & & \\
\hline \multicolumn{9}{|l|}{ Health and birth experience } \\
\hline PROMIS Global-10 scale ${ }^{*}$ & $\sqrt{ }$ & $\sqrt{ }$ & & & $\sqrt{ }$ & & $\sqrt{ }$ & \\
\hline Birth Satisfaction Scale-Revised ${ }^{*}$ & & & $\sqrt{ }$ & & & & & \\
\hline \multicolumn{9}{|l|}{ Mental wellness } \\
\hline Patient Health Questionaire-2 ${ }^{*}$ & $\sqrt{ }$ & $\sqrt{ }$ & & $\sqrt{ }$ & $\sqrt{ }$ & & & \\
\hline Edinburgh Postnatal Depression Scale & $\sqrt{ }$ & $\sqrt{ }$ & & $\sqrt{ }$ & $\sqrt{ }$ & & & \\
\hline \multicolumn{9}{|l|}{ Nutrition } \\
\hline Breastfeeding success & & & $\sqrt{ }$ & $\sqrt{ }$ & $\sqrt{ }$ & & & \\
\hline Breastfeeding confidence & & & $\sqrt{ }$ & & & & & \\
\hline Breastfeeding Self-Efficacy Score-Short Form ${ }^{*}$ & & & $\sqrt{ }$ & $\sqrt{ }$ & $\sqrt{ }$ & & & \\
\hline Infant Feeding Questionnaire & & & & & & & $\sqrt{ }$ & \\
\hline Maternal and child weight/height & $\sqrt{ }$ & $\sqrt{ }$ & $\sqrt{ }$ & $\sqrt{ }$ & $\sqrt{ }$ & $\sqrt{ }$ & $\sqrt{ }$ & $\sqrt{ }$ \\
\hline \multicolumn{9}{|l|}{ Parenting self-efficacy } \\
\hline TOPSE $^{*}$ & & & & $\sqrt{ }$ & $\sqrt{ }$ & $\sqrt{ }$ & $\sqrt{ }$ & $\sqrt{ }$ \\
\hline Parental Sense of Competence Scale ${ }^{*}$ & & & & $\sqrt{ }$ & $\sqrt{ }$ & $\sqrt{ }$ & $\sqrt{ }$ & $\sqrt{ }$ \\
\hline \multicolumn{9}{|l|}{ Medical records retrieval } \\
\hline Obstetric and delivery outcomes & & & $\sqrt{ }$ & & & & & \\
\hline
\end{tabular}

*From the International Consortium for Health Outcomes Measurement (ICHOM) Standard Set of Outcome Measures for Pregnancy and Childbirth PROMIS Patient-Reported Outcomes Measurement Information System, TOPSE Tool to measure Parenting Self-Efficacy

related to antenatal care, breastfeeding, infant care, child growth and development, and postpartum care. These messages are targeted and time specific according to stages in the pregnancy and early childhood. There were designed by the study team's health experts as reference to the Ready4K text messaging programme [31], serving as nudges to influence behaviour and guide first-time families in their pregnancy and early parenting journey. In order to create an interactive and sharing environment with peer support, participants are enrolled automatically to a private social media platform group, where they are encouraged to share information, post photos and interact with each other. More detailed and updated health information related to maternity care and parenting skills are available in the social media platform to provide guidance and support to the participants, as well as to encourage self-learning. Meanwhile, participants can post any query on health concern in their first 1000 days, which will be addressed by the study team's experts. This is a restricted and byinvitation-only social media forum. The contents are monitored by the research staff to ensure this sharing platform contains trustworthy information. We envision that motivated participants will be empowered and eventually, emerge as chat group leaders in this platform.

In Arm 3, we conduct midwife-led continuity care involving individual teleconferencing sessions. Participants will be encountered by midwives who interact with them at various time points throughout the study. During pregnancy, one session that lasts for $15-20 \mathrm{~min}$ is held in the antenatal clinic per trimester. Any questions pertaining to pregnancy or childbirth can be clarified with the midwife. During post-delivery, the same midwife will conduct individualised teleconferencing sessions at 1and 2-week postpartum, and call to check for progression at 6-week, 3- and 6-month postpartum. Participants can discuss any concern relating to postpartum health, infant care, breastfeeding, child growth and development with the midwife. In the case when there is uncertainty about queries asked by the participant, advice from the study team's health experts will be sought. We envision that motivated participants will be empowered and eventually, emerge as support volunteers assisting in midwifery care delivery in the community. 


\section{Outcome measures}

We quantify the impact of interventions by measuring PSE in first-time mothers, along with specific health outcomes.

\section{Primary outcome}

The primary outcome of this RCT is PSE, measured using the Tool to measure Parenting Self-Efficacy (TOPSE) and the Parental Sense of Competence Scale (PSOC) at 6-week as the primary endpoint, followed by 6-, 12-, 18- and 24-month post-delivery. We include both TOPSE and PSOC, as the combination use of these two tools provides a comprehensive measure on the domain-specific and domain-general self-efficacy components. In addition, we are able to evaluate, following suggestions by Bandura [36], any differences in employing two assessment strategies operating at different levels of specificity [8].

The TOPSE is a multidimensional, self-report measure designed to assess parents' perceived ability to parent their children, which took into account the views and experience of parents from a range of cultural and social backgrounds $[37,38]$. The theoretical underpinning of TOPSE is based on self-efficacy theory developed by Bandura [36, 39]. It consists of 48 items which are divided into eight subsections: (1) emotion and affection, (2) play and enjoyment, (3) empathy and understanding, (4) control, (5) discipline and setting boundaries, (6) pressures, (7) self-acceptance and (8) learning and knowledge. Participants will be requested to rate items using a 11-point Likert scale ranging from 0 (completely disagree) to 10 (completely agree). The higher the score, the higher the level of PSE.

The PSOC is a self-report instrument designed to measure parenting satisfaction and efficacy [40]. It is a domain-general measure with items describing common parental ideas regardless of the child's age and the specific tasks that the parent has to face [40]. Although the PSOC has mainly been used in parents with children, it has been found to be valid for use in parents with infants [41, 42]. It consists of 17 items scored on a 6-point Likert scale ranging from 1 (strongly disagree) to 6 (strongly agree). The higher the score, the higher the level of parenting satisfaction and self-efficacy.

\section{Secondary outcomes}

Secondary outcomes include health and birth experience, mental wellness and nutritional outcomes, assessed using validated patient reported outcome measurements, including the use of the International Consortium for Health Outcomes Measurement (ICHOM) Standard Set of Outcome Measures for Pregnancy and Childbirth [43] and by anthropometric measures. Time points of assessment and ICHOM tools are indicated in Table 1.
Health experience will be assessed using the Patient Reported Outcomes Measurement Information System (PROMIS) Global-10 [44]. The instrument consists of 10 items representing general health, physical health, mental health, social health, pain, fatigue, and overall perceived quality of life. Nine of the 10 items are scored on a 5-point Likert scale ranging from 1 (poor/not at all) to 5 (excellent/completely). The pain - 10th item, is scored from 0 (no pain) to 10 (worst imaginable pain). This instrument has been found valid to be used for maternity population [45].

Birth experience will be assessed using the Birth Satisfaction Scale-Revised [46]. The instrument measures women's perceptions on quality of care provision, women's personal attributes and stress experienced during labour. It consists of 10 items rated on a 5-point Likert scale ranging from 0 (strongly disagree) to 4 (strongly agree).

Mental wellness will be assessed using the Patient Health Questionnaire-2 (PHQ-2) and the Edinburg Postnatal Depression Scale (EPDS). The PHQ-2 consists of 2 items rated on a 4-point Likert scale from 0 (not all all) to 3 (nearly every day), to screen for depression as a first step approach [47]. EPDS will be used when a woman scores a three or above on the PHQ-2, in order to further assess depressive symptoms in details. Although EPDS was originally designed to measure postnatal depression [48], it has been shown to be valid in assessing antenatal depression [49]. It consists of 10 items rated on a 4-point Likert scale, ranging from 0 (as much as I always could) to 3 (not at all).

Nutritional outcomes are assessed based on breastfeeding and infant feeding practices, as well as anthropometric measures. We evaluate breastfeeding in terms of breastfeeding success, breastfeeding confidence and breastfeeding self-efficacy. Breastfeeding success is measured according to feeding mode in the past 7 days, with options of 'only breast milk', 'combination of breast milk, formula and/or water', and 'only formula, water or other liquids but not breast milk' [50]. Breastfeeding confidence is evaluated based on a 5-point Likert scale from 1 (not at all) to 5 (very confident) [50]. Breastfeeding Self-Efficacy Score-Short Form is used to assess maternal confidence in her ability to breastfeed her new baby [51]. The instrument consists of 14 items rated on a 5-point Likert scale, ranging from 1 (not at all) to 5 (very confident). The Infant Feeding Questionnaire (IFQ) is used to assess maternal feeding beliefs and practices [52]. This tool has been shown to be valid for use in our population [53]. IFQ is a 28 -item questionnaire rated on a 5-point Likert scale, ranging from 1 (never/disagree a lot) to 5 (always/agree a lot). Nutritional status for both mother and child is assessed throughout the study period, by evaluating maternal weight status across 
pregnancy and during postpartum, and child growth over the first 2 years, i.e. weight and length.

\section{Sample size}

Based on a previous parenting programme in childhood, a moderate effect $(d=0.4)$ on PSE was reported [54]. We postulate that with our early interventions, a difference of $d=0.35$ in the overall TOPSE (primary outcome) score between intervention and control arms will be detected. In order to achieve a power of at least $80 \%$ with a two-sided significance level at 5\%, a total of 150 participants are required for each arm. Assuming a dropout rate of $66 \%$ during the first 1000 -day follow-up, the necessary sample size is 250 participants per arm, resulting in a total of 750 participants for three arms.

\section{Statistical analysis}

One-way analysis of variance (ANOVA) and chi-square tests or Fisher's exact tests will be used to compare differences in baseline characteristics among the three arms. The primary analysis will be according to the intention-to-treat principle, i.e. all participants who were assigned to a condition. Differences in outcomes across three arms at each time-point will be compared using the one-way analysis of covariance (ANCOVA) (General Linear Model, GLM), adjusting for baseline characteristics and measures. Bonferroni correction will be performed for pair-wise comparisons. Linear mixed models will be used to compare the outcomes across time points among the three arms, adjusting for baseline characteristics and measures. Additionally, a priori sensitivity analysis will be conducted for complete cases, i.e. participants with complete follow-up and data. There are no formal planned interim analyses of the primary outcome, but progress reports on all data issues will be presented to investigators of the study team. The level of statistical significance will be set at 0.05 (two-tailed) and 95\% confidence intervals will be calculated.

\section{Quality control}

Research staff who are responsible to recruit and follow-up participants received training on recruitment approach, informed consent taking, randomisation procedure, online questionnaires management, mobile messages (nudges) and reminders deliveries. Midwives received special training on midwifery continuous care service. Monthly meetings will be held with investigators and/or project manager to review study procedures, recruitment strategy, participants' feedback and collected data. An annual report on study progress will be prepared. The study will be audited by an independent party annually.

\section{Data monitoring}

All data will be anonymised. Participants' identifiers will be kept separately in a password-protected file. Electronic data will be managed using an online data capture tool. We will set up a data monitoring team to perform data checking on the completeness, errors and outliers. The staff will perform data checking weekly to ensure questionnaires are submitted by the participants within the 2-week timeframe. To facilitate the data monitoring procedure, a reminder message to check on the data completeness will be triggered automatically by the system after the initial receipt of online questionnaires. If the participants do not submit the questionnaires or with any incomplete section/missing data, research staff will contact the participants to remind or check on the problems faced. A meeting will be held with investigators quarterly to review the data with issues. Paper documents will be kept in a locked cabinet and electronic data will be stored on password-protected computers or hard-disk drives which can only be accessed by research team members. All records will be maintained for at least 6 years after completing the study.

\section{Patient and public involvement}

The research questions, study design, exposure and outcome measures were determined based on a discussion with health experts (clinicians, nurses and researchers) in maternal and child care. Although participants did not directly contribute to the development of research questions and the study design, their needs, preferences and participation burden were considered throughout the process. The results of the study will be disseminated to participants at their request, after the study is completed.

\section{Ethics and dissemination}

Participants will sign a written informed consent after full disclosure and explanation of the study purpose and procedure. In the event of any new information or change in the study protocol that may be relevant to their willingness to continue the study, research staff will contact the participants in timely manner to seek for further consents if required. Participants will be informed that the use of nudges, social media and community midwife care are not yet proven to be a standard care for first-time parents from pregnancy to 2 years post-delivery. The use of these approaches is solely for research purpose. We expect no adverse health effect from the interventions.

The main findings of this study will be published in peer-reviewed international journals. Presentations of the study results will also be carried out in symposia, seminars and conferences. If the interventions are found 
to produce a positive impact, we will consider a media release to publicise the research findings.

\section{Discussion}

This protocol describes the rationale and design of a three-arm RCT that aims to improve PSE and health outcomes of mother-child for first-time families in Singapore, through a choice architecture approach using behavioural nudges and midwife-led care continuity services during the first 1000 days. The interventions are supported by social media platform, individualised teleconferencing sessions and health care expert guidance to create an interactive, sharing and self-learning environment in the community. The study framework is designed by tapping into existing scientific and medical evidence to guide first-time parents to shape their health behaviour since the onset of pregnancy and to improve self-efficacy in early parenting journey. It translates $\mathrm{DOHaD}$ science into advanced disease prevention by intervening at critical windows-the first 1000 days, for optimal promotion of healthy childhood development, and to reduce mental disorders or metabolic diseases in the mother-offspring.

CRADLE is an implementation research, which will contribute to build an evidence-based parenting support system in the community for new parents and new families. Through this study, we will have a better understanding on the needs and ways to well prepare our families for parenting in the first 1000 days, which will impact our population health in long run. Findings from the study will also help us to increase knowledge and experience about effective strategies to promote mental wellbeing and optimal nutrition for pregnant women, postpartum women and young children. This contributes to an effort in breaking intergenerational vicious cycle of chronic disease, which is a current worldwide issue [55]. Besides, the study has the potential to provide insights to develop a sustainable model of health guidance for first-time families in early parenthood, algorithms in mother-child care, and personalised care paths for distinct subsets of first-time families with specific individualised needs in the first 1000 days.

The greatest strength of this study is the use of an integrated choice architecture approach to test out its effect on PSE using a RCT design through the first 1000 days. Studies in health and education provide evidence that text messaging as nudging tool can be an effective way to change complex, continuous and long-term behaviours, underscoring its potential promote parental involvement [33, 35]. The focus on early life intervention starting since early pregnancy enables us to examine the influences of intervention which is expected to be more effective compared to other RCTs on parenting care that only initiated at post-delivery $[30,56]$. Assessments of outcome measures at multiple time-point allow us to monitor and track the health progress longitudinally. Based on the findings, subsequent plans will be considered to examine the feasibility of utilising ICHOM tools and implementing them nationwide to monitor public health in Singapore. We are therefore embarking on a proof-of-concept and proof-of-value study, which is important to address challenges of the know-do gap in real-world setting.

Few limitations are acknowledged. First, the study is restricted by its external validity as all participants will be recruited from one hospital in Singapore. Thus, caution is required to extrapolate the findings to the general population. Nevertheless, KKH houses the largest public maternity unit in Singapore, and manages $\sim 30 \%$ of all live births in Singapore, across a wide sociodemographic spectrum. We will check for generalisability of findings by comparing differences in basic demographic data between this study and other studies involving larger population of pregnant women in Singapore [57]. Second, the study will only involve participants who are able to understand English, which could limit the generalisability of results to other individuals/families who are non-English speaking. However, English is the first language in Singapore which is widely used and understandable across nations, especially the young who are our primarily samples. Third, there may be a risk of crosscontamination between study arms due to the single hospital setting in the recruitment though different antenatal clinics will be involved. In order to reduce the contamination risk, participants will be instructed not to share their information received with others, except partner and members within the social media platform (Arm 2). Finally, we will target only for mothers but not fathers for the measurement outcomes in this study. This is to reduce variations attributable to differential parental responses arise from differences in paternal and maternal expectations. However, the intervention's content and context were designed to be applicable for first-time families, i.e. both parents.

\section{Trial status}

Recruitment for the trial has begun on 15 June 2020 and is estimated to complete on 30 September 2022. The current protocol is version 3, 7 July 2020.

\section{Abbreviations}

CRADLE: Community-enabled Readiness for 1000 Days Learning Ecosystem; DOHaD: Developmental Origins of Health and Disease; EPDS: Edinburg Postnatal Depression Scale; ICHOM: International Consortium for Health Outcomes Measurement; KKH: KK Women's and Children's Hospital; PHQ-

2: Patient Health Questionnaire-2; PSE: Parenting self-efficacy; PSOC: Parental Sense of Competence Scale; TOPSE: Tool to measure Parenting Self-Efficacy; RCT: Randomised controlled trial 


\section{Supplementary Information}

The online version contains supplementary material available at https://doi. org/10.1186/s13063-021-05144-5.

Additional file 1. SPIRIT 2013 Checklist. Recommended items to address in a clinical trial protocol and related documents.

\section{Acknowledgements}

We thank the CRADLE study group, including Lai Yun Ho, Derrick Chan, Mei Chien Chua, Gaik Nai Ng, Serene Thain, Kok Hian Tan, Nur Adila Binte Ahmad Hatib, Sing Zhi Kee and Jing Wen Ng.

\section{Authors' contributions}

The concept for the study and its design was originated by KCN, T and OMC, with important inputs into the study design, protocol, standard operating procedures and delivery of the programmes by SLL, JT, SWC and NKAR. KCN is the principal investigator of the study. T, NRAR and OMC are the coinvestigators. SLL drafted the manuscript. All authors reviewed and approved the final manuscript.

\section{Funding}

This research is supported by the Tanoto Foundation (02/FY2019/EX/14-A51). The funder has no role in study design, data collection and analysis, decision to publish or preparation of the manuscript.

\section{Availability of data and materials}

This manuscript does not contain any data. Datasets during and/or analysed during the study will be available from the principal investigator (KCN) on reasonable request. The results of the study will be submitted for publication in peer-reviewed journals as soon as possible after analysis.

\section{Declarations}

\section{Ethics approval and consent to participate}

Ethical approval has been granted by the Centralised Institutional Review Board of SingHealth (reference 2019/2781). We perform the study in accordance with the Declaration of Helsinki. Written informed consent will be obtained from participants before baseline assessment and group assignment. Before providing the consent, participants will be explained and given information on the objectives and characteristics of the study. They will also be informed that participation in the study is voluntary, that they can choose to withdraw at any time, and are guaranteed to continue to receive the standard routine care regardless of study participation.

\section{Consent for publication}

Not applicable.

\section{Competing interests}

The authors declare that they have no competing interests.

\section{Author details}

'Department of Reproductive Medicine, KK Women's and Children's Hospital, 100 Bukit Timah Road, Singapore 229899, Singapore. ²Duke-NUS Medical School, 8 College Road, Singapore 169857, Singapore. ${ }^{3}$ Singapore Institute for Clinical Sciences, Agency for Science, Technology and Research (A*STAR), 30 Medical Drive, Singapore 117609, Singapore. ${ }^{4}$ Division of Nursing, KK Women's and Children's Hospital, Singapore, 100 Bukit Timah Road, Singapore 229899, Singapore. ${ }^{5}$ Medical Board, KK Women's and Children's Hospital, 100 Bukit Timah Road, Singapore 229899, Singapore. ${ }^{6}$ Department of Paediatrics, KK Women's and Children's Hospital, 100 Bukit Timah Road, Singapore 229899, Singapore. ${ }^{7}$ Yong Loo Lin School of Medicine, National University of Singapore, National University Health System, Singapore 119228, Singapore. ${ }^{8}$ Lee Kong Chian School of Medicine, Nanyang Technological University, 11 Mandalay Road, Singapore 308232, Singapore.
Received: 29 April 2020 Accepted: 22 February 2021

Published online: 06 March 2021

\section{References}

1. Bateson P, Barker D, Clutton-Brock T, Deb D, D'Udine B, Foley RA, et al. Developmental plasticity and human health. Nature. 2004:430:419-21.

2. Hanson M, Godfrey KM, Lillycrop KA, Burdge GC, Gluckman PD. Developmental plasticity and developmental origins of non-communicable disease: theoretical considerations and epigenetic mechanisms. Prog Biophys Mol Biol. 2011;106:272-80.

3. Suzuki K. The developing world of DOHaD. J Dev Orig Health Dis. 2018;3: 266-9.

4. Koletzko B, Godfrey KM, Poston L, Szajewska H, Van Goudoever JB, de Waard $M$, et al. Nutrition during pregnancy, lactation and early childhood and its implications for maternal and long-term child health: the early nutrition project recommendations. Ann Nutr Metab. 2019;2:93-106.

5. 1,000 days. Why 1,000 days. 2020. https://thousanddays.org. Accessed 20 Mar 2020

6. Borrelli SE, Walsh D, Spiby H. First-time mothers' expectations of the unknown territory of childbirth: uncertainties, coping strategies and 'going with the flow'. Midwifery. 2018;63:39-45.

7. Wernand JJ, Kunseler FC, Oosterman M, Beekman AT, Schuengel C. Prenatal changes in parenting self-efficacy: linkages with anxiety and depressive symptoms in primiparous women. Infant Ment Health J. 2014;35:42-50.

8. Wittkowski A, Garrett C, Calam R, Weisberg D. Self-report measures of parental self-efficacy: a systematic review of the current literature. J Child Fam Stud. 2017;26:2960-78.

9. Albanese AM, Russo GR, Geller PA. The role of parental self-efficacy in parent and child well-being: a systematic review of associated outcomes. Child Care Health Dev. 2019;45:333-63.

10. Glatz T, Buchanan CM. Over-time associations among parental self-efficacy, promotive parenting practices, and adolescents' externalizing behaviors. J Fam Psychol. 2015:29:427.

11. Jones TL, Prinz RJ. Potential roles of parental self-efficacy in parent and child adjustment: a review. Clin Psychol Rev. 2005;25:341-63.

12. Abdollahi F, Agajani-Delavar M, Zarghami M, Lye MS. Postpartum mental health in first-time mothers: a cohort study. Iran J Psychiatry Behav Sci. 2016;10:e426.

13. Gross $\mathrm{CL}$, Marcussen K. Postpartum depression in mothers and fathers: the role of parenting efficacy expectations during the transition to parenthood. Sex Roles. 2017;76:290-305.

14. Secer Z, Ogelman G. Analysing Mothers' self-efficacy perception towards parenting in relation to peer relationships of 5-6 year-old preschool children. Educ Sci Theory Pract. 2012:12:2001-8.

15. Ahun MN, Consoli A, Pingault JB, Falissard B, Battaglia M, Boivin M, et al. Maternal depression symptoms and internalising problems in the offspring: the role of maternal and family factors. Eur Child Adolesc Psychiatry. 2018; 27:921-32.

16. Heerman WJ, Taylor JL, Wallston KA, Barkin SL. Parenting self-efficacy, parent depression, and healthy childhood behaviors in a low-income minority population: a cross-sectional analysis. Matern Child Health J. 2017;21:115665.

17. Bandura A. The nature and structure of self-efficacy. In: Self-efficacy: the exercise of control. New York: WH Freeman and Company; 1997. p. 37-78.

18. Bryanton J, Gagnon AJ, Hatem M, Johnston C. Predictors of early parenting self-efficacy: results of a prospective cohort study. Nurs Res. 2008;57:252-9.

19. Morse CA, Buist A, Durkin S. First-time parenthood: influences on pre-and postnatal adjustment in fathers and mothers. J Psychosom Obstet Gynaecol. 2000;21:109-20

20. Mihelic M, Filus A, Morawaska A. Correlates of prenatal parenting expectations in new mothers: is better self-efficacy a potential target for preventing postnatal adjustment difficulties? Prev Sci. 2016;17:949-59.

21. Soltani F, Maleki A, Shobeiri F, Shamsaei F, Ahmadi F, Roshanaei G. The limbo of motherhood: women's experiences of major challenges to cope with the first pregnancy. Midwifery. 2017;55:38-44.

22. Ong SF, Chan WC, Shorey S, Chong YS, Klainin-Yobas P, He HG. Postnatal experiences and support needs of first-time mothers in Singapore: a descriptive qualitative study. Midwifery. 2014:30:772-8.

23. Nakamura $Y$, Takeishi Y, Ito N, Ito M, Atogami F, Yoshizawa T. Comfort with motherhood in late pregnancy facilitates maternal role attainment in early postpartum. Tohoku J Exp Med. 2015;235:53-9. 
24. Norling-Gustafsson A, Skaghammar K, Adolfsson A. Expectant parents' experiences of parental education within the antenatal health service. Psychol Res Behav Manag. 2011:4:159.

25. Dahlen HG, Barclay LM, Homer CS. The novice birthing: theorising first-time mothers' experiences of birth at home and in hospital in Australia. Midwifery. 2010;26:53-63.

26. Barimani M, Frykedal KF, Rosander M, Berlin A. Childbirth and parenting preparation in antenatal classes. Midwifery. 2018:57:1-7.

27. Svensson J, Barclay L, Cooke M. Randomised-controlled trial of two antenatal education programmes. Midwifery. 2009;25:114-25.

28. Sandall J, Soltani H, Gates S, Shennan A, Devane D. Midwife-led continuity models versus other models of care for childbearing women. Cochrane Database Syst Rev. 2016;4:CD004667.

29. Toohill J, Callander E, Gamble J, Creedy DK, Fenwick J. A cost effectiveness analysis of midwife psycho-education for fearful pregnant women-a health system perspective for the antenatal period. BMC Pregnancy Childbirth. 2017;17:217

30. Jiao N, Zhu L, Chong YS, Chan WC, Luo N, Wang W, et al. Web-based versus home-based postnatal psychoeducational interventions for first-time mothers: a randomised controlled trial. Int J Nurs Stud. 2019;99:103385

31. ParentPowered. Ready4K. 2018. https://ready4k.parentpowered.com/. Accessed 17 Mar 2020.

32. Blumenthal-Barby J, Opel DJ. Nudge or grudge? Choice architecture and parental decision-making. Hast Cent Rep. 2018;48:33-9.

33. York BN, Loeb S. One step at a time: the effects of an early literacy text messaging program for parents of preschoolers: National Bureau of Economic Research; 2014. http://www.nber.org/papers/w20659. Assessed 20 Mar 2020

34. Thaler RH, Sunstein CR. Nudge: improving decisions about health, wealth, and happiness. New Haven: Yale University Press; 2008. https://yalebooks.ya le.edu/book/9780300122237/nudge. Assessed 7 Apr 2020

35. Hansen PG, Jespersen AM. Nudge and the manipulation of choice: a framework for the responsible use of the nudge approach to behaviour change in public policy. Euro J Risk Regul. 2013;4:3-28.

36. Bandura A. Regulation of cognitive processes through perceived selfefficacy. Dev Psychol. 1989;25:729-35.

37. Kendall S, Bloomfield L. Developing and validating a tool to measure parenting self-efficacy. J Adv Nurs. 2005:51:174-81.

38. Bloomfield $L$, Kendall S. Parenting self-efficacy, parenting stress and child behaviour before and after a parenting programme. Prim Health Care Res. 2012;13:364-72

39. Bandura A. Self-efficacy mechanism in human agency. Am Psychol. 1982;37: 122-47.

40. Johnston C, Mash EJ. A measure of parenting satisfaction and efficacy. J Clin Child Psychol. 1989;18:167-75.

41. Chung FF, Wan GH, Kuo SC, Lin KC, Liu HE. Mother-infant interaction quality and sense of parenting competence at six months postpartum for first-time mothers in Taiwan: a multiple time series design. BMC Pregnancy Childbirth. 2018;18:365

42. Gilmore L, Cuskelly M. Factor structure of the parenting sense of competence scale using a normative sample. Child Care Health Dev. 2009; 35:48-55.

43. ICHOM (International Consortium for Health Outcomes Measurement) Pregnancy and childbirth: the standard set. https://www.ichom.org/ portfolio/pregnancy-and-childbirth/. Accessed 26 Oct 2020

44. PROMIS Health Organization and PROMIS Cooperation Group. PROMIS Global 10. 2020. https://www.codetechnology.com/promis-global-10/. Accessed 24 Mar 2020

45. Slavin V, Gamble J, Creedy DK, Fenwick J, Pallant J. Measuring physical and mental health during pregnancy and postpartum in an Australian childbearing population - validation of the PROMIS Global Short Form. BMC Pregnancy Childbirth. 2019;19:370.

46. Hollins Martin CJ, Martin CR. Development and psychometric properties of the birth satisfaction scale-revised (BSS-R). Midwifery. 2014;30:610-9.

47. Kroenke K, Spitzer RL, Williams JB. The Patient Health Questionnaire-2: validity of a two-item depression screener. Med Care. 2003:41:1284-94.

48. Cox JL, Holden JM, Sagovsky R. Detection of postnatal depression. Development of the 10-item Edinburgh Postnatal Depression Scale. Br J Psychiatry. 1987;150:782-6.

49. Kozinszky Z, Dudas RB. Validation studies of the Edinburgh Postnatal Depression Scale for the antenatal period. J Affect Disord. 2015;176:95-105.
50. Nijagal MA, Wissig S, Stowell C, Olson E, Amer-Wahlin I, Bonsel G, et al. Standardized outcome measures for pregnancy and childbirth, an ICHOM proposal. BMC Health Serv Res. 2018;18:953.

51. Dennis CL. The breastfeeding self-efficacy scale: psychometric assessment of the short form. J Obstet Gynecol Neonatal Nurs. 2003;32:734-44.

52. Baughcum AE, Powers SW, Johnson SB, Chamberlin LA, Deeks CM, Jain A, et al. Maternal feeding practices and beliefs and their relationships to overweight in early childhood. J Dev Behav Pediatr. 2001;22:391-408.

53. Quah PL, Cheng TS, Cheung YB, Yap F, Saw SM, Godfrey KM, et al. Maternal and infant correlates of maternal feeding beliefs and practices in a multiethnic Asian population: the GUSTO (Growing Up in Singapore Towards healthy Outcomes) study. Public Health Nutr. 2016;19:2789-98.

54. Ulfsdotter $M$, Enebrink $P$, Lindberg L. Effectiveness of a universal healthpromoting parenting program: a randomized waitlist controlled trial of All Children in Focus. BMC Public Health. 2014;14:1083.

55. World Health Organization. Global action plan for the prevention and control of noncommunicable diseases 2013-2020. 2013. https://apps.who. int/iris/bitstream/handle/10665/94384/9789244506233_rus.pdf. Accessed 7 Apr 2020.

56. Kristensen $\mathrm{H}$, Kronborg $\mathrm{H}$. What are the effects of supporting early parenting by enhancing parents' understanding of the infant? Study protocol for a cluster-randomized community-based trial of the Newborn Behavioral Observation (NBO) method. BMC Public Health. 2018;18:832.

57. Soh SE, Tint MT, Gluckman PD, Godfrey KM, Rifkin-Graboi A, Chan YH, et al. Cohort profile: Growing Up in Singapore Towards healthy Outcomes (GUSTO) birth cohort study. Int J Epidemiol. 2014:43:1401-9.

\section{Publisher's Note}

Springer Nature remains neutral with regard to jurisdictional claims in published maps and institutional affiliations.

Ready to submit your research? Choose BMC and benefit from:

- fast, convenient online submission

- thorough peer review by experienced researchers in your field

- rapid publication on acceptance

- support for research data, including large and complex data types

- gold Open Access which fosters wider collaboration and increased citations

- maximum visibility for your research: over $100 \mathrm{M}$ website views per year

At $\mathrm{BMC}$, research is always in progress.

Learn more biomedcentral.com/submissions 